\title{
Temperature influence on the diethylamine sensing abilities of CuO nanoparticles deposited by atmospheric pressure plasma
}

\author{
G. Filipič ${ }^{1}$ and J. Gruenwald ${ }^{2}$ \\ ${ }^{1}$ Jozef Stefan Institute, Jamova cesta 39, 1000 Ljubljana, Slovenia \\ ${ }^{2}$ Gruenwald Laboratories, Taxberg 50, 5660 Taxenbach, Austria
}

(Received: 13. Dec. 2019, Accepted: 10. April 2020, Published online: 10. April 2020)

In this work, we present a copper oxide nanostructured analysed as a gas sensor, but the focus of the paper is on the temperature dependence of the sensor sensing properties. As a case study, temperature dependent diethylamine sensing is presented. The CuO nanoflakes were deposited and evenly distributed on intercalated electrodes by an atmospheric pressure plasma source. The sensor was electrically connected to ohmmetre and inserted in an oven chamber where it was isolated from the atmosphere and heated to the desired temperatures. The intrinsic resistance of the sensor was measured in dependence of the temperature and the temperature change rate. Then the possibility to detect diethylamine was investigated. Furthermore, the amine influence on the sensor resistance in the correlation to the amine effect on the sensor temperature was shown. Finally, the temperature dependence of the amine detection was explored. It was demonstrated that the plasma deposited nanostructures could be a way towards developing reliable sensing of the amine at low temperatures of $100{ }^{\circ} \mathrm{C}$ and below.

DOI: 10.31281/jtsp.v1i1.10

gregor.filipic@guest.arnes.si

\section{Introduction}

Metal-oxide semiconductors are a popular choice for chemical sensors due to the ease of manufacturing, structure, and morphology manipulations they offer. In the case of gas sensing, common materials are $\mathrm{SnO}_{2}[1,2,3]$, $\mathrm{ZnO}[4,5,6], \ln _{2} \mathrm{O}_{3}[7,8,9]$ and $\mathrm{CuO}[10,11,12]$. However, they come with the downside of not being energy efficient due to the need of heating them to a working temperature that can be quite high, even above $300^{\circ} \mathrm{C}[13]$ Despite many attempts to lower the needed temperature and the availability of some sensors operating at room temperature $[14,15]$, this remains a pertinent issue. This paper aims at obtaining a better understanding of the temperature dependence of the resistive type of gas sensor. As a case study, we have chosen a copper-oxide nanomaterial to detect diethylamine. The ability to detect amines even in low concentrations is of high importance due to health hazards, which are connected to these organic compounds. It was found, for example, that diethylamine (DEA), which is used in the industrial production of rubber, pharmaceuticals or pesticides, is responsible for excessive bone growth (hyperostosis) in the nasal cavities (turbinates) of lab mice [16] as well as for irritation of the nose and eyes [17]. These negative effects on the health were shown for DEA concentrations down to some ppm. The prepared sensor was first exposed to heating to gain an insight into its resistance dependence to temperature and checked for the repeatability of the process. Then we analysed the response - change of its resistance - to the
DEA injection and the desorption process. The temperature was simultaneously measured to detect any anomalies in the sensing due to possible temperature perturbation. Finally, we have repeated the detection experiments of DEA at different temperatures of the sensor.

\section{Experimental Setup}

The gas sensing surface was homogeneously covered with CuO nanoflakes that were deposited on intercalated gold electrode substrates with a non-thermal plasma jet at atmospheric pressure, which is described in detail elsewhere $[18,19]$. The depositions with the atmospheric pressure plasma source were conducted with a mixture of highly pure 5.0 gases; $1000 \mathrm{sccm}$ of Ar and $7000 \mathrm{sccm}$ of $\mathrm{N}_{2}$ gas flow. The input power was $150 \mathrm{~W}$ fixed at $30 \mathrm{~mA}$ input current. The copper was introduced into the plasma via a sacrificial electrode made of Cu with 99.99 purity. The plasma source was fastened in a vertical position, and samples were exposed for deposition $6 \mathrm{~mm}$ underneath the nozzle. The morphology of the deposited material is seen in Fig. 1. The CuO grows in the form of thin flakes $(\mathrm{d}<10 \mathrm{~nm})$. The hight of the accumulated flake layer is roughly $1 \mu \mathrm{m}$.

As it is known from a previous study regarding the chemical compositions of these depositions, the nanoparticles consist of $\mathrm{CuO}$ on top of a $\mathrm{Cu}_{2} \mathrm{O}$ layer [19]. The analysis of the chemical variety of copper oxide in the aforementioned paper was done by depth profile measurements with XPS. The nanoflakes are homogeneously distributed 


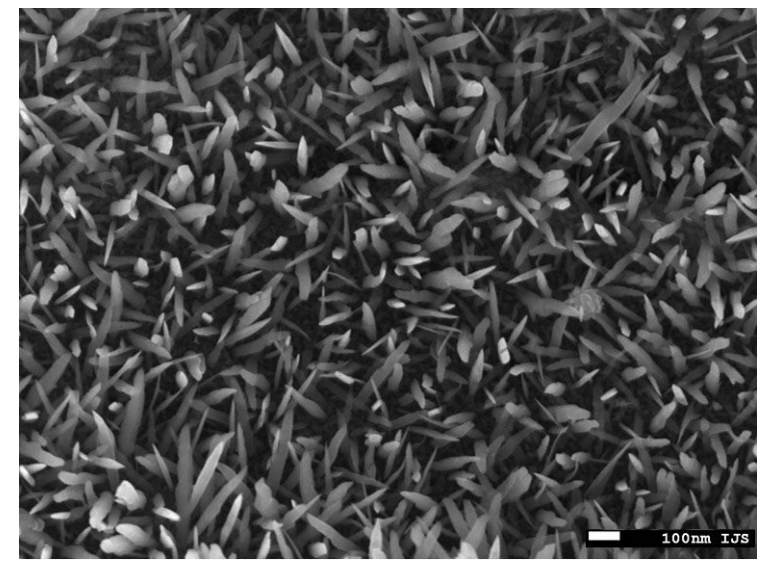

Figure 1: Secondary electrons (SE) SEM image of the nanostructured $\mathrm{CuO}$ semiconductor surface.

over the copper and gold substrates used in our experiments (Fig. 2). The large area that is achieved with such a kind of nanoparticles enables excellent gas sensing properties in general.

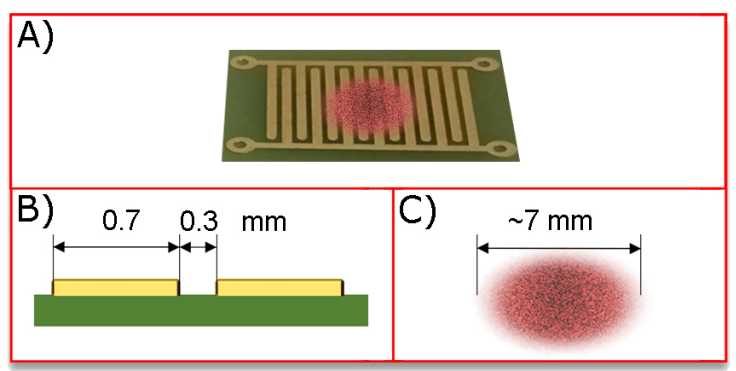

Figure 2: Simple schematic of the sensor chip; A) the intercalated gold electrode on a polymer substrate with a representation of nanomaterial deposition; $B$ ) the size of the electrodes and the space between them in $\mathrm{mm}$; C) the approximate circular size of the deposited material in $\mathrm{mm}$.

The gas sensing experiments were conducted at a low pressure in a vacuum system to control the sensor environment and for the safety of the researchers. The experimental setup was the same throughout all of the sensing experiments: sensor was electrically connected to a 2450 Keithley source-metre and inserted into a centre of a $1 \mathrm{~m}$ long quartz tube with an $80 \mathrm{~mm}$ ODE in a vacuum furnace with $45 \mathrm{~cm}$ heating zone (OTF-1200X-II, MTI Corp.). A thermocouple was inserted in the tube from the opposite side, $2 \mathrm{~cm}$ from the gas sensor, also in the axis of the tube - to monitor the temperature in the vicinity of the sensor. The voltage on the thermocouple was recorded by the same source-metre, and it was translated to temperature, $\mathrm{T}$, in ${ }^{\circ} \mathrm{C}$ via:

$$
T=-\frac{V}{41 \cdot 10^{-6}}+21
$$

The chamber was evacuated and kept at low pressure by a rotary pump. The pump was able to reduce the pressure in the chamber down to $2.5 \mathrm{~Pa}$. Dur- ing the amine sensing experiments, the amine was injected into the chamber from the opposite side by utilising a Bronkhorst's vaporising system (CEM W-102A). The vaporising system comprises two inlets, one for liquid (amine) that is introduced under pressure through a liquid mass-flow-metre, and another for carrying gas (argon), which is injected through a mass-flow controller. The gas and the liquid are mixed in the vaporiser, and the vapour is introduced to the vacuum chamber.

The first sets of experiments, results shown in sections III.a) and III.b), were performed to test the reaction of the sensor resistance to temperature change. The sensor was in a vacuum while the furnace was set to heat up from room temperature to $100{ }^{\circ} \mathrm{C}$, stay there for $10 \mathrm{~min}$, and then naturally cool down. The experiment was repeated three times. The following part of the experiment - results presented in section III.c) - was performed to determine the sensor response to a DEA injection into the system. In parallel, the influence of amine on the temperature of the sensor was monitored by thermocouple. The sensor was put in the chamber, which was again evacuated to $2.5 \mathrm{~Pa}$, heated to $100{ }^{\circ} \mathrm{C}$, and left there for the sensor to thermalise. Then, DEA was injected into the system with a rate of $250 \mathrm{sccm}$ carried by $300 \mathrm{sccm}$ of Ar. The changes in the resistance of the sensor and temperature of the thermocouple were measured against the time. They were continued to be monitored for a couple of minutes after the flow of amine was switched off. In the last experiment - III.d) DEA response at different temperatures was evaluated. Thus, the same experiment as in III.C) was repeated with several cycles of the DEA injection/evacuation at different set temperatures: $100,115,130$, and $145^{\circ} \mathrm{C}$.

\section{Experimental Results}

\section{III.a) Resistance change due to temperature change}

The experimental results regarding the connection between temperature and response of the $\mathrm{CuO}$ sensors are presented and discussed. First, the measurements of the resistance of the sensor during heating and cooling are shown in Fig. 3. There have been three cycles of heating and cooling done in the vacuum. The resistance curve during the heating in the first cycle, $M 1$, is noticeably different from the other two measurements. The assumption is that this is due to the slow desorption of various substances that were adsorbed on the surface during the sensor shelf-time, which affects the conductivity of the surface. The measurements M2 and M3 were done after finishing with M1 without exposing the sensor to the atmosphere. Their curves are almost identical. Another thing one can notice is that during the heating the resistance is proportional to the inverse Boltzmann factor, which is to be expected since resistance is inverse proportional to the density number of the charge carriers of a semiconductor: 


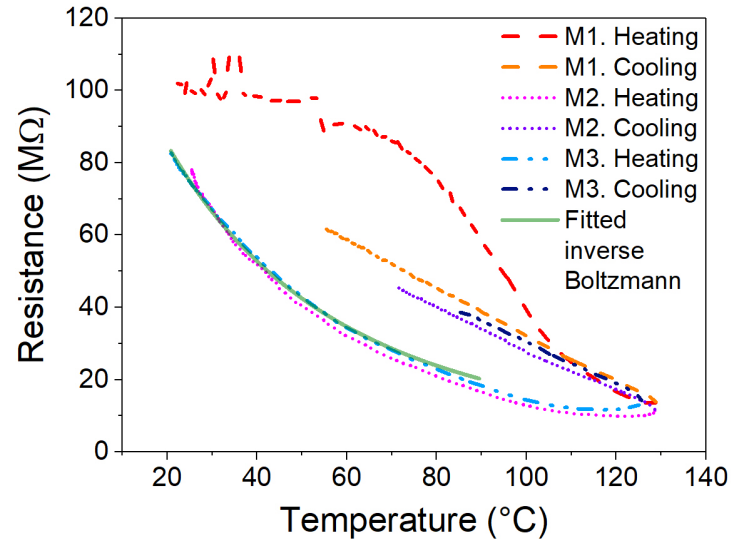

Figure 3: Resistance of the sensor measured during heating and cooling. The measurements were done three times.

$$
n_{0}=2\left(\frac{m^{*} k T}{2 \pi \hbar^{2}}\right)^{3 / 2} \exp \left(-\frac{\left(E_{c}-E_{f}\right)}{k T}\right)
$$

The resistance dependence on the temperature during cooling is quite linear as it can also be noticed from the graph in Fig. 3.

\section{III.b) Varying heating-cooling rates}

We have conducted another experiment with different rates of heating and cooling. We started at around $1.1{ }^{\circ} \mathrm{C} / \mathrm{min}$ heating, then increased the heating to $4.2^{\circ} \mathrm{C} / \mathrm{min}$ until reaching the maximum temperature of about $100{ }^{\circ} \mathrm{C}$. The cooling has started with $3.3^{\circ} \mathrm{C} / \mathrm{min}$ decrease and finished with a linear estimation of around $1{ }^{\circ} \mathrm{C} / \mathrm{min}$ cooling. The results are depicted in Fig. 4; first, the temperature and resistance change with time are presented in $4 \mathrm{~A}$ and then combined into a resistance change against temperature plot in $4 \mathrm{~B}$. It is observed that different rates of temperature change yield different responses.

At small temperature changes, it seems the change of the resistance with the temperature could be linearly fitted, but with different slopes for the different rates of the temperature change. Furthermore, even in the MaxwellBoltzmann distribution fit, the parameters would have significantly changed between the different rates of the temperature changes. Further research is needed to fully understand these dependencies, while it is clear that the sensor's resistance can be easily detected already at $50{ }^{\circ} \mathrm{C}$.

\section{III.c) Sensor case study}

For a better response, the choice to heat the sensor to $100{ }^{\circ} \mathrm{C}$ was made. First, the sensor was inserted into the chamber close to the thermocouple, as in the previous experiments. The chamber was evacuated to the base pressure $(2.5 \mathrm{~Pa})$ and heated to the pre-set temperature. After
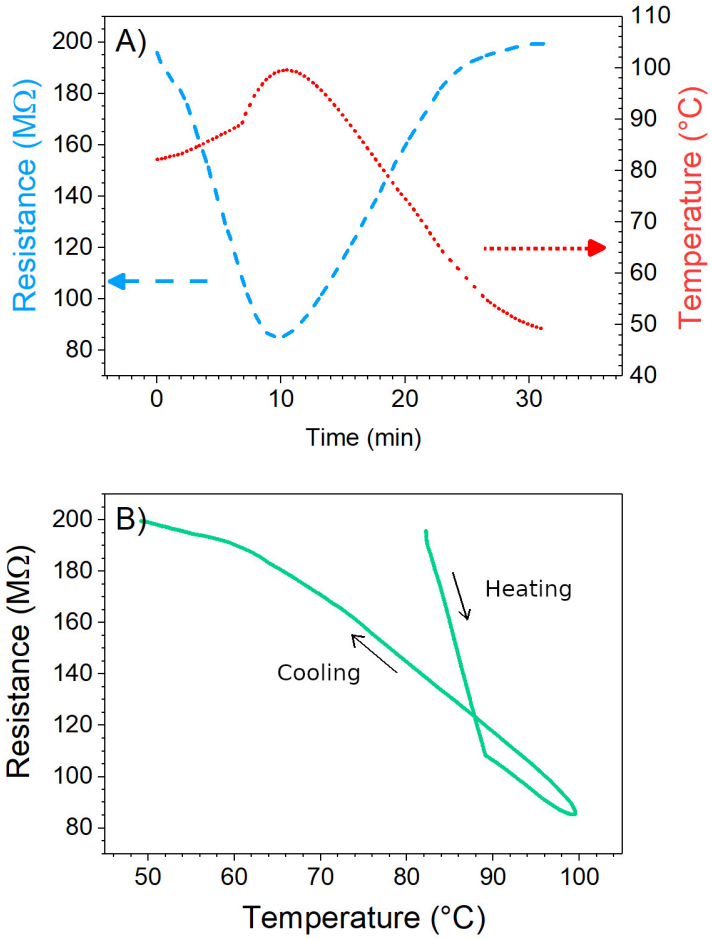

Figure 4: Influence of different rates of temperature change on the resistance of the sensor: A) measurement of resistance and temperature against time;

B) presentation of the resistance against temperature.

the sensor got thermalised, Ar was leaked into the system with $300 \mathrm{sccm}$ of flow, while still pumping the chamber. After the thermalisation, the DEA was injected into the system with $250 \mathrm{sccm}$ - the pressure rose to about $70 \mathrm{~Pa}$. After $10 \mathrm{~min}$, the DEA flow was terminated, and the desorption started. The resistance of the sensor and the temperature measured with the thermocouple are presented in Fig. 5.

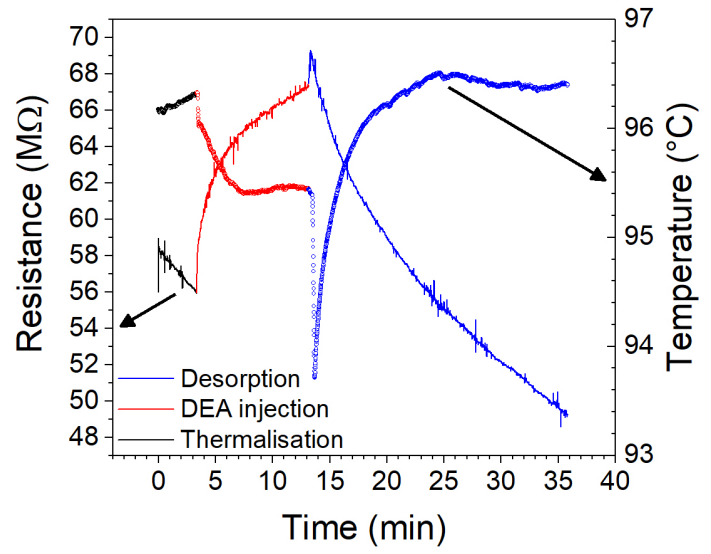

Figure 5: Graphs of the sensor resistance and the thermocouple temperature before (black), during (red), and after the DEA injection of the system (blue).

It can be seen that after the DEA injection, the tempera- 
ture of the thermocouple is dropping. This is ascribed to the cooling by the evaporating DEA. The temperature is falling until the equilibrium between the DEA adsorption and desorption is reached, and thermodynamic equilibrium to furnace heating is established. In this process, the temperature changes for about $1{ }^{\circ} \mathrm{C}$. During this time the resistance increases for around $9 \mathrm{M} \Omega$. The resistance is increasing fast after the DEA hits the surface of the sensor and continues rising even after the temperature is in equilibrium. In the beginning stage, one cannot decouple the temperature effect from the sensing response in the initial phase. However, the cooling for about $1{ }^{\circ} \mathrm{C}$ at the fastest rate in Fig. 4 caused only about $5 \mathrm{M} \Omega$ resistance increase. In this case, the fastest rate contributed less than $0.5{ }^{\circ} \mathrm{C}$, which would contribute only $2.5 \mathrm{M} \Omega$ to the resistance increase according to Fig. 4 . In the later phase, while the temperature is constant, it is clear that the resistance increases due to DEA adsorption on the surface. The time during the slow drop of the temperature then contributed to the $6.5 \mathrm{M} \Omega$ resistance increase, which clearly can not be all attributed to the temperature change. Furthermore, the resistance curve shape follows the path towards a saturated value, which is expected. After the DEA source is closed, a fast evaporation phase follows. The resistance temporary increases in a jump before it starts to decrease steadily. The jump can be explained by rapid desorption, which cools the surface as seen from the temperature curve. As it is evident from Fig. 4, fast changes in temperature yield more significant changes in resistance; since the temperature dropped in an almost discrete jump, the resistance of the sensor is increased. When the temperature starts slowly rising, the resistance change is governed mostly by the amount of DEA adsorbed on the surface and its desorption. In the last part of the experiment, the temperature is equalised again. Since the sensor resistance did not reach the saturation level in the $10 \mathrm{~min}$ in the DEA atmosphere, the sensing can be done again even before the complete amine desorption.

\section{III.d) Sensor response at different tempera- tures}

As the pre-tests were successful, the testing of the sensor for the detection of DEA at different temperatures was interesting. The known experiment for sensing the DEA was repeated at $100,115,130$, and $145^{\circ} \mathrm{C}$; at each temperature two cycles were run, each with $10 \mathrm{~min}$ amine flow and 20 min desorption. The results are shown in Fig. 6 as the change in resistance from the initial value before the DEA injection.

The sensor response function is calculated as a relative change of resistance presented in Eq.3:

$$
S=\frac{R-R_{0}}{R_{0}}
$$

The measurements show that the resistance increases for between 4 and $6 \mathrm{M} \Omega$ in the first $10 \mathrm{~s}$ and between 6 and 8 in the first $20 \mathrm{~s}$. As we saw in the previous chapter, less than $3 \mathrm{M} \Omega$ can be caused purely by the cooling effect. Thus, the sensor response after the first 10 seconds can

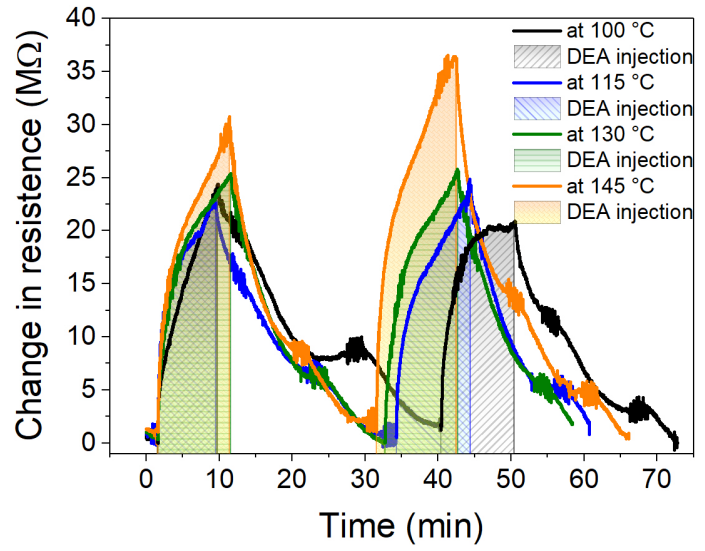

Figure 6: Graph of change in resistance of the sensor at four different temperatures during two cycles of amine (DEA) injections and desorption. The DEA injection periods are noted by the colouring of the graphs under the curves.

be already used for the estimation of the vapour concentration based on the measuring of the transient response parameters [20]. Also at much longer times, longer than 5 mins, the temperature effect on the response can also be seen from the Fig. 7 - higher the temperature of the sensor, the higher the response is. Even more so, the response dependence on temperature is not linear. Returning to the number density of charge carriers at a specific temperature and the Eq. 2, one can again expect an exponential dependence of the response to the same concentration of the analyte at different temperatures. This was confirmed by this experiment and is depicted in Fig. 7 where the maximum responses after 10 min of sensor exposure to DEA are plotted against temperatures together with an exponential fit to guide the eye. The saturation level of the resistance was not observed - the sensor response and recovery times are well above $5 \mathrm{~min}$. However, one needs to keep in mind that we were operating the sensor at very low temperatures and that if the semiconductor sensors temperature is ramped up to $250{ }^{\circ} \mathrm{C}$ and above the response and recovery times become much shorter [21].

\section{Conclusions}

We have deposited copper oxide nanoflakes with a large surface area on intercalated electrodes and shown that it is possible to utilise it to sense diethylamine. The sensor response research was done at low pressure to determine intrinsic sensor properties. It was presented that the resistance of the sensor changes with the temperature obeying exponential temperature dependence. Furthermore, the results are reproducible if the temperature is changed in the same manner in all cases. However, if the rate of temperature change is varied, also the resistance dependence on temperature changes. This phenomenon needs further examination in order to evaluate the sensor operation in a possible temperature fluctuating environment. Furthermore, this work illuminates 


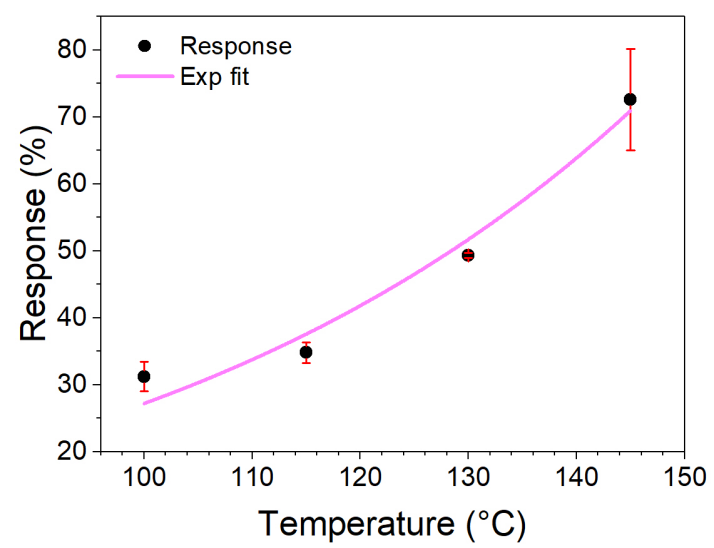

Figure 7: The sensor response to amine injection at different temperatures and the exponential fit with temperature in the denominator of the exponent. The error bars represent the difference in the response between the two cycles at each temperature. The flow of DEA was 250 and $\operatorname{Ar} 300$ sccm, respectively.

the sensor resistance response during the initial contact of the vapour of the detection interests with the surface of the sensor. In this phase, the temperature drops very fast, which can have an influence on the resistance, as seen in the DEA detection experiment. The same behaviour needs to be tested in the live-world conditions at atmospheric pressure, and higher temperatures to estimate if these results need to be taken into account when trying to use sensors transient times for the analyte concentration detection. In addition, there is a curious sudden resistance spike at the time of DEA switch off, which was denoted to rapid DEA desorption from the surface in the low-pressure atmosphere and consequent temporary cooling of the sensor. By demonstrating the ability of CuO nanoflakes to respond to DEA even at low temperatures, it was also possible to indirectly prove that atmospheric pressure plasma sources can be successfully used as a tool for gas sensor fabrication. Since the plasma deposition occurs at ambient pressure directly on conducting substrates, it is a very efficient fabrication technology. In the future, the sensor interaction with different atmospheres (e.g. water vapour, oxygen) will be studied to find how these gases influence the sensing possibilities and temperature dependencies.

\section{References}

[1] B. Wang, L. Zhu, Y. Yang, N. Xu, and G. Yang, "Fabrication of a $\mathrm{SnO}_{2}$ nanowire gas sensor and sensor performance for hydrogen," The Journal of Physical Chemistry C, vol. 112, no. 17, pp. 6643-6647, 2008.

[2] H. Huang, Y. Lee, O. Tan, W. Zhou, N. Peng, and $\mathrm{Q}$. Zhang, "High sensitivity $\mathrm{SnO}_{2}$ single-nanorod sensors for the detection of $\mathrm{H}_{2}$ gas at low temperature," Nanotechnology, vol. 20, no. 11, p. 115501, 2009.

[3] H. Huang, H. Gong, C. L. Chow, J. Guo, T. J. White, M. S. Tse, and O. K. Tan, "Low-temperature growth of $\mathrm{SnO}_{2}$ nanorod arrays and tunable n-p-n sensing response of a $\mathrm{ZnO} / \mathrm{SnO}_{2}$ heterojunction for exclusive hydrogen sensors," Advanced Functional Materials, vol. 21, no. 14, pp. 2680-2686, 2011.

[4] O. Lupan, G. Chai, and L. Chow, "Novel hydrogen gas sensor based on single $\mathrm{ZnO}$ nanorod," Microelectronic Engineering, vol. 85, no. 11, pp. 2220-2225, 2008.

[5] J. J. Hassan, M. A. Mahdi, C. W. Chin, H. Abu-Hassan, and Z. Hassan, "A high-sensitivity room-temperature hydrogen gas sensor based on oblique and vertical ZnO nanorod arrays," Sensors and Actuators B: Chemical, vol. 176, pp. 360-367, 2013.

[6] L. Wang, Y. Kang, X. Liu, S. Zhang, W. Huang, and S. Wang, "ZnO nanorod gas sensor for ethanol detection," Sensors and Actuators B: Chemical, vol. 162, no. 1, pp. 237-243, 2012.

[7] M. Kaur, N. Jain, K. Sharma, S. Bhattacharya, M. Roy, A. Tyagi, S. Gupta, and J. Yakhmi, "Roomtemperature $\mathrm{H}_{2} \mathrm{~S}$ gas sensing at ppb level by single crystal $\ln _{2} \mathrm{O}_{3}$ whiskers," Sensors and Actuators $B$ : Chemical, vol. 133, no. 2, pp. 456-461, 2008.

[8] W. Zang, Y. Nie, D. Zhu, P. Deng, L. Xing, and $X$. Xue, “Core-shell $\operatorname{In}_{2} \mathrm{O}_{3} \mathrm{ZnO}$ nanoarray nanogenerator as a self-powered active gas sensor with high $\mathrm{H}_{2} \mathrm{~S}$ sensitivity and selectivity at room temperature," The Journal of Physical Chemistry C, vol. 118, no. 17, pp. 9209-9216, 2014.

[9] S. Elouali, L. G. Bloor, R. Binions, I. P. Parkin, C. J. Carmalt, and J. A. Darr, "Gas sensing with nanoindium oxides (In2O3) prepared via continuous hydrothermal flow synthesis," Langmuir, vol. 28, no. 3, pp. 1879-1885, 2012.

[10] A. Ghosh, A. Maity, R. Banerjee, and S. Majumder, "Volatile organic compound sensing using copper oxide thin films: addressing the cross sensitivity issue," Journal of Alloys and Compounds, vol. 692, pp. 108-118, 2017.

[11] O. Alev, E. Şennik, and Z. Z. Öztürk, "Improved gas sensing performance of $\mathrm{p}$-copper oxide thin film $/ \mathrm{n}$ $\mathrm{TiO}_{2}$ nanotubes heterostructure," Journal of Alloys and Compounds, vol. 749, pp. 221-228, 2018.

[12] S. Steinhauer, A. Köck, C. Gspan, W. Grogger, L. Vandamme, and D. Pogany, "Low-frequency noise characterization of single $\mathrm{CuO}$ nanowire gas sensor devices," Applied Physics Letters, vol. 107, no. 12, p. 123112, 2015.

[13] V. Kruefu, A. Wisitsoraat, A. Tuantranont, and S. Phanichphant, "Ultra-sensitive $\mathrm{H}_{2} \mathrm{~S}$ sensors based on hydrothermal/impregnation-made Ru-functionalized $\mathrm{WO}_{3}$ nanorods," Sensors and Actuators B: Chemical, vol. 215, pp. 630 - 636, 2015.

[14] S. Bhuvaneshwari, S. Papachan, and N. Gopalakrishnan, "Free standing $\mathrm{CuO}-\mathrm{MnO}_{2}$ nanocomposite for room temperature ammonia sensing," AIP Conference Proceedings, vol. 1832, no. 1, p. 050126, 2017.

[15] T. M. Perfecto, C. A. Zito, and D. P. Volanti, “Design of nanostructured $\mathrm{WO}_{3} \cdot 0.33 \mathrm{H}_{2} \mathrm{O}$ via combination 
of ultrasonic spray nozzle and microwave-assisted hydrothermal methods for enhancing isopropanol gas sensing at room temperature," CrystEngComm, vol. 19, pp. 2733-2738, 2017. Design of nanostructured $\mathrm{WO}_{3} \cdot 0.33 \mathrm{H}_{2} \mathrm{O}$ via.

[16] R. L. Grant, S. O. Taiwo, and D. McCant, "Assessment of chronic inhalation non-cancer toxicity for diethylamine," Inhalation toxicology, vol. 27, no. 14, pp. 778-786, 2015.

[17] G. R. Lundqvist, M. Yamagiwa, O. F. Pedersen, and G. D. Nielsen, "Inhalation of diethylamine - acute nasal effects and subjective response," American Industrial Hygiene Association Journal, vol. 53, no. 3, pp. 181-185, 1992.

[18] J. Gruenwald, K. Fricke, M. Fröhlich, J. Kolb, and M. Polak, "Deposition of copper oxide coatings with an atmospheric pressure plasma source: Characterization of the plasma and films II: The films," submitted to Plasma Processes Polym., 2016.

[19] J. Gruenwald, K. Fricke, M. Fröhlich, J. Kolb, and M. Polak, "Deposition of copper oxide coatings with an atmospheric pressure plasma source: Characterization of the plasma and films I: The plasma," submitted to Plasma Processes Polym., 2016.

[20] P. F. Satterthwaite, S. Eberle, S. Nedelcu, C. Roman, and C. Hierold, "Transient and steady-state readout of nanowire gas sensors in the presence of lowfrequency noise," Sensors and Actuators B: Chemical, vol. 297, p. 126674, oct 2019.

[21] M.-R. Wu, W.-Z. Li, C.-Y. Tung, C.-Y. Huang, Y.-H. Chiang, P.-L. Liu, and R.-H. Horng, "NO gas sensor based on $\mathrm{ZnGa}_{2} \mathrm{O}_{4}$ epilayer grown by metalorganic chemical vapor deposition," Scientific Reports, vol. 9, may 2019.

\section{(1)}

Open Access This article is licensed under a Creative Commons Attribution 4.0 International License, which permits use, sharing, adaptation, distribution and reproduction in any medium or format, as long as you give appropriate credit to the original author(s) and the source, provide a link to the Creative Commons license, and indicate if changes were made. The images or other third party material in this article are included in the articles Creative Commons license, unless indicated otherwise in a credit line to the material. If material is not included in the article's Creative Commons license and your intended use is not permitted by statutory regulation or exceeds the permitted use, you will need to obtain permission directly from the copyright holder. To view a copy of this license, visit: http://creativecommons.org/licenses/by/4.0/. 